\title{
Advances in medical technology and its impact on health care in developing countries
}

\section{Editorial}

Advanced technology is applied in many areas of healthcare for diagnosis as well as for therapy. Medical Technology is the use of scientific knowledge to improve the health care by new equipment to make work easier, pleasant, quick and productive. Now, in modern hospitals, technologists and biomedical engineers are involved in routine day to day work, research and technical development. As a result, the close association between these professionals has strongly influenced the modern health care facilities and resulted in many state of art technology to be incorporated into the health care system. Health care facilities in coming years will witness greater contribution of biomedical engineers to make it more effective and quality based.

Evolving technology has long been a major tool for the clinicians. Instruments based on the basic principles of physics have been used for years in diagnosis and treatment. Sphygmomanometer (BP apparatus), thermometer, stethoscope and microscope are some of the common instruments used in medicine extensively for long time. Advanced technologies in medicine like computed tomography, Ultrasound diagnostic devices, radio nuclear imaging, radiation therapy, lithotripsy, dialysis, ventilators, etc have produced a sea change in medical practice, taking us from conventional patient care to highly adaptive treatment capable of challenging many dreaded diseases.

The technological advances in diagnosis, surgical procedures, drug delivery, etc, have made life comfortable as well as complicated also. Management and treatment of diseases has become largely dependent on discoveries in newer drugs, surgical techniques, diagnostic and therapeutic equipment. It is also true that availability of treatment options of many diseases have increased the life expectancy. However, many new diseases have also cropped up, the population of old people has increased and therefore, the challenges associated with geriatric population must be addressed.

Technology in medicine could be compared to a double edged sword which must be used judiciously depending upon the need and socioeconomic factors. Use of technology in medicine should not be fashionable and one should not enter in rat race of getting new technology until it is required. Technology comes at a price, heavy dependence on technology increases medical expenses and therefore, it may be out of the reach of the common man. Heavy dependence on modern medicines is taking us away from healthier and natural lifestyles. Technology also brings unhealthy competition among pharmaceutical companies and medical equipment manufacturers to provide something new and start marketing by offering lucrative packages to health professionals which may not be beneficial to the patients. Technology also gives options which may lead to confusions and conflicts.

Health care in developing countries is becoming expensive and out of reach of common persons. Developing countries are blindly adopting developed countries model of health care without taking into account socioeconomic conditions and life style considerations.
Volume 2 Issue 2 - 2017

\author{
Lalit Mohan Aggarwal \\ Department of Radiotherapy \& Radiation Medicine, Banaras \\ Hindu University, India
}

Correspondence: Lalit Mohan Aggarwal, Department of Radiotherapy \& Radiation Medicine, Institute of Medical Sciences, Banaras Hindu University, Varanasi (UP)-221 005, Tel 09336936073, Email Imaggarwal@yahoo.com

Received: February 25, 2017| Published: February 27, 2017

Developing countries have limited budgets for high end medical equipment therefore, they cannot afford to have hi-tech five-star hospitals and interventions for the time being. These facilities are for rich people and their percentage is very little. Instead of going for high end medical facilities, developing countries must strengthen primary health care infrastructure. They must concentrate on prevention of diseases by providing safe drinking water for the masses, three square meals a day, uncontaminated and a clean environment for dwelling. Sanitation with a stress on sewerage disposal and toilet facilities in villages and slums of large cities are the urgent needs. It is not wise in blindly accepting a new, expensive therapy until or unless it is cost effective and serves the masses. Politicians and bureaucrats who are responsible for providing health care to the citizens do not have much trust on their healthcare systems and go to developed countries for their own treatment.

Health care has rich tradition of evidence-based practice but in recent years it has become increasingly absorbed with technological advances. This increased emphasis on technology has escalated the cost of medical treatment and health care has become the subject of the corporate sector than the state. The implementation of advanced technologies often leads to less personal contact between the physician and the patient.

The actual application of advanced medical equipment raises questions about cost, efficacy and ethics. The high initial investment and operating costs in health sector is a big challenge for developing countries. Technology has the advantage of saving time but it requires well-qualified medical/paramedical staff to manage this sophisticated equipment which may otherwise not give desirable outcome. Therefore, the major challenges for using technically advanced equipment in developing countries along with cost are appropriate human resources, qualified and trained personnel for their optimum use. Advanced technological needs for health care must be considered in the context of the needs of the developing countries in terms of essential infrastructure, human resource and types of prevailing diseases so that whole population could be benefitted. 
The Medical science of tomorrow would demand a more intense scientific approach, and newer ways of thinking for better health care. Impact of technology should be seen in totality than in isolation. Good health depends on life style, environment, hygiene, quality of food, water, etc. Prevention is better than cure. But modern life style has moved us away from prevention and we are highly dependent on technology, which is not a healthy trend. Modern life style has become disease proliferator which has resulted in big business for equipment and pharmaceutical companies. The introduction of any new technology in medicine must provide better health care and improved quality of life at affordable expenses without losing human touch.

\section{Acknowledgements}

None.

\section{Conflict of interest}

Author declares that there is no conflict of interest. 